\title{
FAST Hue-PrESERVING HistogRaM EQUALIZATION METHODS FOR COLOR IMAGE CONTRAST ENHANCEMENT
}

\author{
David Menotti ${ }^{1,2,4}$, Laurent Najman ${ }^{2}$, Jacques Facon ${ }^{3}$ and Arnaldo de A. Albuquerque ${ }^{4}$ \\ ${ }^{1}$ Computing Department, Federal University of Ouro Preto, Ouro Preto, Brazil \\ menotti@iceb.ufop.br \\ ${ }^{2}$ Equipe A3SI, ESIEE Paris, Université Paris-Est, Noisy-le-Grand, France \\ 1.najman@esiee.fr \\ ${ }^{3}$ PPGIA, Pontifícia Universidade Católica do Paraná, Curitiba, Brazil \\ faconeppgia.pucpr.br \\ ${ }^{4}$ Computing Department, Universidade Federal de Minas Gerais, Belo Horizonte, Brazil \\ arnaldoedcc.ufmg.br
}

\begin{abstract}
In this work, we formalize a generic fast hue-preserving histogram equalization method based on the RGB color space for image contrast enhancement and two versions of that generic process. The first method estimates a RGB 3D histogram to be equalized using R-red, G-green, and B-blue 1D histograms, while the second method employs RG, RB, and GB 2D histograms. The histogram equalization is performed using shift hue-preserving transformations, avoiding unrealistic colors. Our methods have linear time and space complexities with respect to the size of the image and do not need to apply conversions from a color space to another in order to perform the image enhancement. Such design complies with real-time applications requirements. An objective assessment comparing our methods and others is performed using a contrast measure and a color image quality measure, where the quality is established as a weighting of the naturalness and colorfulness indexes. We analyze 300 images from the dataset of the University of Berkeley. Experiments show that the value of the image contrast produced by our methods is in average $50 \%$ greater than the original image value, keeping the quality of the produced images close to the original one.
\end{abstract}

\section{KEYWORDS}

Histogram equalization; contrast enhancement; hue-preserving; color image; RGB color space.

\section{INTRODUCTION}

The integration of cameras in portable devices, especially in mobile phones and PDAs, has increased the need for image processing software which can work fast, use as little memory as possible and produce good quality images. Having this scenario in mind, many algorithms have been produced in order to enhance the images obtained by these devices, including $[1,2,3,4,5,6,7,8]$.

A great variety of methods were developed for gray-level contrast image enhancement. Among these methods are the ones based on histogram equalization. Histogram Equalization (HE) [9] methods generate a uniform histogram (i.e., uniform distribution) from an original image by using the entire range of discrete levels of the image and stretching and/or redistributing these levels in 
the output histogram. The extension of these methods to color images is not straightforward. This is because there are some particular properties of color images which need to be properly taken into account during image enhancement.

Among these properties are the luminance, saturation and hue attributes of the color [10]. Color spaces such as $H S V, H S I, C I E L U V, C I E L A B$ were conceived based on these three attributes. Whereas the luminance represents the achromatic part of the color (e.g., it can be defined as a weighted function of the $R, G$, and $B$ color channels), the saturation and hue refer to the chromatic part of the image. The saturation can be defined as the measure of the amount of white in the color, and the hue as the color attribute which decides its real meaning ("real color"), e.g., red or green. In the context of color image enhancement, the hue should not be changed for any pixel such that an unnatural aspect for the output images is avoided.

On the other hand the $R G B$ color space (i.e., $R$-red, $G$-green, and $B$-blue) is commonly used for displaying, transmitting, and storing color images in digital devices, such as mobile phones, cameras and PDAs. For image processing tasks, this color space is not the most appropriated one, since other color spaces explicitly separate the meaning of the attribute colors while the RGB one does not. It is trivial the conversion from the $R G B$ color space to a Luminance-Hue-Saturation $(L H S)$-based color space. However, this process can be both not appropriate to the device where the images are acquired and not suitable for real-time applications. Moreover, tackling the wellknown gamut problem when working on a $L H S$-based color space is required [11].

The literature of HE methods for color contrast image enhancement present works based on the $R G B, L H S$, and CIELUV color spaces, as described. In [12], it is proposed a HE method based on the brightness, saturation, and hue $(H S I)$ color space. This technique yields an image with uniform brightness and saturation histograms, leaving the hue channel unchanged. In [13], a technique similar to this one is proposed. In this latter work, a special care is given to saturation, where high frequency information is present. However, portable devices require more efficient methods to be used in.

Furthermore, in [14], a gray-level images enhancement method based on semi-HE and a modified cosine function is proposed, and for color images by equalizing the three channels $(R, G$, and $B)$ separately, an extension of this methodology is suggested. Although this method is fast, it does not preserve the hue. An algorithm for HE taking into account color channels correlation through mesh deformation is also proposed in [15]. This method always generates almost (i.e. discrete) uniform color histograms and optimally uses the color space. It is indicated for scientific visualization purposes, but not for image enhancement, since it does not preserve the image natural features. In [3], a novel dynamic enhanced algorithm based on histogram specification is proposed to enhance the contrast of the gray-level images without losing the original histogram characteristics. However, when the method is extended to the color space, first the image is converted to a $L S H$-based color space and after only the luminance is enhanced, keeping both the hue and saturation unchanged.

By analysing the methods previously presented, we can observe that none of them present all the characteristics required for use in portable devices: to improve the images contrast, be fast, and still preserve the hue. In order to reach such aim, this work formalizes a generic fast huepreserving HE method based on the $R G B$ color space for image contrast enhancement. From the generic method we extrapolate two variants, which are determined by the histograms dimension used, i.e., $1 D$ or $2 D$. Indeed, in $[16,17]$, the methods presented here were previously introduced. The method presented in [16] is not hue-preserving. However, with the modifications presented here it is. Hue-preserving transformations directly on the $R G B$ color space [11] perform the histogram equalization, avoiding the conversion requirement between color spaces and the gamut problem, keeping the hue unchanged. Besides, contrast image enhancement (i.e., the 
improvement of the variance on the luminance attribute) is produced by our methods, and, at the same time, from the equalization of the uniform $R G B$ histogram, the saturation is modified. Using $R, G$ and $B 1 D$ histograms and $R G, R B$, and $G B 2 D$ histograms, respectively, the methods estimate the $R G B 3 D$ histogram to be equalized, yielding algorithms with space and time complexities linear with respect to the size of the image. Having these features, these methods become suitable for real-time applications.

Addition to describing these new fast methods, this article presents another significant contribution. The images processed are evaluated using quantitative measures, allowing us to perform an objective comparison among the presented methods and two other related methods previously introduced in the literature. We run experiments in a dataset of 300 images of the University of Berkeley, analyzing the contrast and the color quality of the original and processed images (guided by naturalness and colorfulness index [18]).

The remainder of this paper is organized as follows. Basic definitions for color images are presented in Section 2. Previous works related to our methods are described in Section 3, and in Section 4 our new methods are presented. Experiments are shown in Section 5 and, finally, conclusions are drawn in Section 6.

\section{BASIC DEFINITIONS}

In a context of discrete variables, the histogram of a variable represents the absolute frequency of each discrete value, whereas the probability density function of a variable constitutes the relative frequency of these values. The probability distribution function (or the cumulative probability density function), in turn, can be seen as the probability of a variable to be less or equal to a value. The estimation of the probability of an event happening is performed using these functions. Considering that a color image is a discrete variable, this section describes its multidimensional histograms and their probability functions, which will be used throughout this work.

Let $\mathbb{N}$ and $\mathbb{Z}$ denote the set of natural and integer numbers, respectively. Let $X$ be a subset of points $(x, y) \in \mathbb{N}^{2}$, such that $0 \leq x<m$, and $0 \leqq y<n$, where $m$ and $n$ denote the dimensions of $X$. Note that $\|X\|=m n$. A mapping $I$, from $X$ to $\mathbb{Z}_{L}^{3}$, is called a (color) image (in the RGB color space). In applications, $L$ is typically 256 . By abuse of terminology we denote a color image by $I^{R G B}$.

Indeed, a color image $I^{R G B}$ has three mappings from $X$ to $\mathbb{Z}_{L}$, which are the red, green and blue images, i.e. $I^{R}, I^{G}$ and $I^{B}$, respectively. Let us also define three other mappings from $X$ to $\mathbb{Z}_{L}^{2}$, i.e., $I^{R G}, I^{R B}$ and $I^{G B}$. We call these mappings red/green, red/blue and green/blue images, respectively.

For a point $(x, y) \in X, R_{i}=I^{R}(x, y), G_{i}=I^{G}(x, y)$ and $B_{i}=I^{B}(x, y)$ are called the red, green and blue levels of the point $(x, y)$ in $I^{R G B}$, respectively, where $0 \leq R_{i}, B_{i}, G_{i}<L$. We can also denote $\left(R_{i}, G_{i}, B_{i}\right),\left(R_{i}, G_{i}\right),\left(R_{i}, B_{i}\right)$ and $\left(G_{i}, B_{i}\right)$ by $I_{(x, y)}{ }^{R G B}, I_{(x, y)}{ }^{R G}, I_{(x, y)}{ }^{R B}$ and $I_{(x, y)}{ }^{G B}$, respectively. In the following, we define $1 D, 2 D$ and $3 D$ histograms and probability density and distribution functions for color images.

For the $1 D$ case, we first consider the $R$ color channel. Let $H_{R i}{ }^{I R}$ be the absolute frequency of level $R_{i}$ in image $I^{R}$, where $0 \leq R_{i}<L$. The mapping $H^{I R}$ from the levels of image $I^{R}$ to its absolute frequency levels, i.e., $H^{I R}: \mathbb{Z}_{L} \rightarrow \mathbb{N}$, is called the histogram of image $I^{R}$. Let $P^{I R}$ be the probability density function of $I^{R}$. We denote by $P_{R i}^{I R}$ the probability of level $R_{i}$, i.e., $P_{R i}^{I R}=H_{R i}{ }^{I R} m n$, where 0 $\Delta R_{i}<L$. Let $C^{I R}$ be the cumulative density function (or the probability distribution function) of $I^{R}$. We denote by $C_{R i}{ }^{I R}$ the cumulative density of level $R_{i}$, i.e., $C_{R i}{ }^{I R}=\sum_{r i=0}{ }_{i}^{R} P_{r i}{ }^{I R}$, where $0 \leq R_{i}<L$. It is immediate to extend the above definitions of $I^{R}$ image, i.e., $H^{I R}, P^{I R}$ and $C^{I R}$, to $I^{G}$ and $I^{B}$ images. 
For the $2 D$ case, we first consider the $R$ and $G$ color channels. Let $H_{R i, G i}{ }^{I R G}$ be the absolute frequency of $R_{i}$ and $G_{i}$ colors in image $I^{R G}$. The mapping $H^{I R G}$ from the levels of image $I^{R G}$ to its absolute frequency levels, i.e., $H^{I R G}: \mathbb{Z}_{L}^{2} \rightarrow \mathbb{N}$, is called the histogram of image $I^{R G}$. Let $P^{I R G}$ be the probability density function of $I^{R G}$. We denote by $P_{R i, G i}^{I R G}$ the probability of $\left(R_{i}, G_{i}\right)$, i.e., $P_{R i, G i}{ }^{I R G}=$ $H_{R i, G i}^{I R G}(m n)$. Let $C^{I R G}$ be the cumulative density function of $I^{R G}$. We denote by $C_{R i, G i}{ }_{I R G}$ the cumulative density of $\left(R_{i}, G_{i}\right)$, i.e., $C_{R i, G i}^{I R G}=\sum_{r i=0}^{R} \sum_{i} \underset{i=0}{G}{ }_{i} P_{r i, g i}{ }_{I R G}$. It is immediate to extend the above definitions of $I^{R G}$ image, i.e., $H^{I R G}, P^{I R G}$ and $C^{I R G}$, to $I^{R B}$ and $I^{G B}$ images.

For the $3 D$ case, let us define a single histogram and probability functions. Let $H_{R i, G i B i}{ }^{I R G B}$ be the absolute frequency of $R_{i}, G_{i}$, and $B_{i}$ colors in image $I$. The mapping $H^{I R G B}$ from the levels of image $I$ to its absolute frequency levels, i.e., $H^{I R G B}: \mathbb{Z}_{L}^{3} \rightarrow \mathbb{N}$, is called the histogram of image $I^{R G B}$. Let $P^{I R G B}$ be the probability density function of $I^{R G B}$. We denote by $P_{R i, G i, B i}{ }^{I R G B}$ the probability of $\left(R_{i}, G_{i}, B_{i}\right)$, i.e., $P_{R i, G i, B i}{ }^{I R G B}=H_{R i, G i, B i}^{I R G B}(m n)$. Let $C^{I R G B}$ be the cumulative density function of $I^{R G B}$. We denote by $C_{R i, G i, B i}{ }^{I R G B}$ the cumulative density of $\left(R_{i}, G_{i}, B_{i}\right)$, i.e., $C_{R i, G i, B i}{ }^{I R G B}=\sum_{r i=0}{ }_{i} \sum_{g}{ }_{i=0}^{G}{ }_{i} \sum$ ${ }_{b i=0}^{B}{ }_{i} P_{r i, g i, b i}{ }^{I R G B}$.

\section{PREVIOUS WORKS}

In this section, we present two HE methods directly related to our proposed methods. Note that all the HE methods described in this article work in three phases: (1) they compute the histogram of the image, (2) they compute the density and distribution probability functions of the image from the histograms, and (3) they enhance the image though HE.

The process carried out to compute the histogram of the image is the same in all methods. With a single scan throughout the image we can compute $1 D, 2 D$, or $3 D$ histograms, according to the definitions given in Section 2.

The second phase, where the density and distribution probability functions are calculated, strongly depends on the dimensions of the probability functions used for the method. It is well known that a typical color image have not its $R, G$, and $B$ color channels neither full correlated nor totally independent distributed. Hence, the dimension (i.e., $1 D, 2 D$, or $3 D$ ) of the density and distribution probability functions of the images used for the methods has a great impact in the quality of enhanced images and in the time complexity of the methods. In this respect, whereas some methods take into account only the red, green and blue channels separately (calculating $1 D$ histograms), others consider the correlation among these channels two at-a-time, or even consider the three of them all together.

Regarding the third phase (the histogram equalization itself), methods can follow very specific rules to achieve it. The classical method processes the $1 D$ histograms separately, and then employs the equalized histograms to enhance the image. Other methods process the image pixel by pixel, using an iterative process, in a way that the histogram of the output enhanced image has a uniform distribution, i.e., it is equalized.

In the next section, the classical HE method for gray-level IE is described, which is extended for color images. We then show the $3 D$ HE method proposed by Trahanias and Venetsanopoulos in [19]. This last method present important concepts which will be then incorporated into our methods, described later on in Section 4.

\subsection{Classical 1D Histogram Equalization}

In this section, the HE method for monochrome images (e.g., gray-level or red ones) is described, followed by its extension to cope with $I^{R G B}$ images. The goal of HE is to uniformly distribute the 
input histogram over the entire range of levels or, equivalently, to generate a cumulative density function which increases monotonically as a straight line, such that an image contrast enhancement is achieved. We focus the description of the method on red images and then extend it to green and blue images. Putting together these definitions in red, green and blue images, we can perform $\mathrm{HE}$ on the $R G B$ color space.

The HE method for red images is described as follows. Let $I$ and $O$ be the original and the equalized images, or the input and the output images, respectively. Let $H^{I R}, P^{I R}$ and $C^{I R}$ be defined as in Section 2. Let $H^{O R}$ be the desired uniform histogram of the output image, where any level $R_{o}$ has the same amount of pixels, i.e., $H_{R o}{ }^{O R}=(m n)$, or the same density, i.e., $P_{R o}{ }^{O R}=1 L$. The cumulative density function $C^{O R}$ is defined in function of $R_{o}$ as $C_{R o}{ }^{O R}=\sum_{r o=0}{ }_{o} P_{r o}{ }^{I R}=\left(R_{o}+1\right) L$.

The $R_{o}^{\prime}$ output equalized level corresponding to the input level $R_{i}$ is obtained as the one that minimizes the difference between $C_{R o}{ }^{\prime} O R$ and $C_{R i}{ }^{I R}$. In other words, the output level $l$ ' for the input level $R_{i}$ can be computed as the transformation function

$T^{I R}\left(R_{i}\right)$, i.e., $R_{o}^{\prime}=T^{I R}\left(R_{i}\right)=\left\langle(L-1) \bar{C}_{R_{i}}^{I^{R}}\right\rangle$ where $\langle z\rangle$ stands for the nearest integer to $z \in \mathbb{R}$. To generate the output enhanced image with this transformation, for any pixel $(x, y) \in X$, we obtain the output value $O^{R}(x, y)$ as $R_{o}^{\prime}=T^{R}\left(R_{i}\right)$, where $R_{i}=I^{R}(x, y)$.

This method can be easily extended for color contrast image enhancement by applying separately the equalization process described above to the $I^{R}, I^{G}$, and $I^{B}$ images, separately. A well-known problem comes with this extended method: since it is not hue preserving [11], it produces unrealistic colors.

Note that this method has $O(\max (\operatorname{mn}, L))$ and $O(L)$ time and space complexities, respectively. From now on, we call this extended method as the classical $1 D$ HE method, i.e., C1DHE method.

\subsection{D Histogram Equalization}

In this section, the method proposed by Trahanias and Venetsanopoulos [19] is described. The correlation of the three channels, $R, G$, and $B$, is taken into account simultaneously in this method.

Let $I$ be the input color image and $O$ be the output color image. Following the definition in Section 2, consider $H^{I R G B}, P^{I R G B}$, and $C^{I R G B}$. For the output image, let $H^{O R G B}$ be the uniform histogram, in which any entry $\left(R_{o}, G_{o}, B_{o}\right)$ has the same amount of pixels, since the same density, i.e., $P_{R o, G o, B o}{ }^{O R G B}=1 L^{3}$ is desired or such output histogram, i.e., $H_{R o, G o, B o}{ }^{O R G B}=(m n)$. Hence, any entry $\left(R_{o}, G_{o}, B_{o}\right)$ in $C^{O R G B}$ is calculated using $P^{O R G B}$, i.e.,

$$
C_{R_{o}, G_{o}, B_{o}}^{R C B}=\frac{\left(R_{o}+1\right)\left(G_{o}+1\right)\left(B_{o}+1\right)}{L^{3}}
$$

To yield the output enhanced image, for any input pixel $(x, y) \in X$, where $\left(R_{i}, G_{i}, B_{i}\right)=I^{R G B}(x, y)$, the smallest $\left(R_{o}, G_{o}, B_{o}\right)$ for which the inequality

$$
C_{R_{i}, G_{i}, B_{i}}^{I R C B}-C_{R_{D}, G_{D}, B_{D}}^{R C B} \geq 0 \text {, }
$$

holds. However, an ambiguity is present in this step of calculating the output histogram, mainly because many possible solutions for $\left(R_{o}, G_{o}, B_{o}\right)$ which satisfy Equation 2 may exist. This ambiguity is remedied as follows. The computed value of $C^{I R G B}$ at $\left(R_{i}, G_{i}, B_{i}\right)$ is initially compared to the value of $C^{O R G B}$ at $\left(R_{o}, G_{o}, B_{o}\right)$. If $C^{I R G B}$ is greater (resp. less) than $C^{O R G B}$, then the indexes $R_{o}$, 
$G_{o}$, and $B_{o}$ are repeatedly increased (resp. decreased), one at-a-time, until Equation 2 is satisfied. The obtained $\left(R_{o}, G_{o}, B_{o}\right)$ is the output entry to the corresponding input $\left(R_{i}, G_{i}, B_{i}\right)$, i.e., if $(x, y) \in X$ and $\left(R_{i}, G_{i}, B_{i}\right)=I^{R G B}(x, y)$, then $O^{R G B}(x, y)=\left(R_{o}, G_{o}, B_{o}\right)$.

From now on, we call the Trahanias and Venetsanopoulos $3 D$ method as TV $3 D H E$ method. The TV3DHE method has $O\left(\max \left(m n L, L^{3}\right)\right)$ and $O\left(L^{3}\right)$ time and space complexities, respectively. Note that the methods discussed in this section have drawbacks that make them not suitable for realworld and real-time applications. Whereas the C1DHE method is not hue-preserving, the TV3DHE method is neither hue-preserving nor complies with real-time application requirements.

\section{FAST HUE-PRESERVING HISTOGRAM EQUALIZATION METHODS FOR COLOR IMAGE CONTRAST ENHANCEMENT}

In this section, we present a generic method which, in contrast with the methods presented in the previous section, is both hue-preserving and has time and space complexities which complies with real-world and real-time applications. We formalize two variants from the generic method, which are determined by the histograms dimension used to estimate the $3 D$ probability functions, i.e., $1 D$ or $2 D$ histograms. In other words, the probability function estimation phase is the variant point of the generic method.

\subsection{Generic Hue-preserving Histogram Equalization Method}

In this section, our generic method is presented. It is, as the other ones, split in three phases. Initially, let $I$ be the input image and $O$ the output image. Let the input $\# D$ histograms and probability functions be defined as in Section 2, where \# is the histogram dimension used (this is the variant point of our method). Although the proposed method work with $\# D$ histograms per say, but a $3 D$ pseudo-histogram, $H^{\prime R G B}$. The $H^{\prime R G B}$ definition is based on a pseudo $3 D$ cumulative density function.

The second phase of our method, the computation of this cumulative density function, $C^{I R G B}$, is performed for any entry $\left(R_{i}, G_{i}, B_{i}\right)$ as the product of the three $\# D$ cumulative functions. The variant methods are formalized in details in Sections 4.2 and 4.3.

The third phase works as follows. The method described in Section 3.2, in order to minimize Equation 2, iteratively increases or decreases the values of $R_{o}, G_{o}$ and $B_{o}$. Here, for any image pixel we propose to find the output triplet $\left(R_{o}, G_{o}, B_{o}\right)$ in a single step, i.e., $O(1)$. Thus, from Equations 1 and 2, we can obtain

$$
C_{R_{i}, G_{i}, B_{i}}^{I_{R C B}}-\frac{\left(R_{o}+1\right)\left(G_{o}+1\right)\left(B_{o}+1\right)}{L^{3}}=0
$$

If we take $R_{o}, G_{o}$ and $B_{o}$ as $R_{i}+k, G_{i}+k$ and $B_{i}+k$, respectively, where $k$ would be the number of iterations required for minimizing Equation 2, we obtain

$$
\begin{aligned}
& k^{3}+ \\
& k^{2}\left[R_{i}^{\prime}+G_{i}^{\prime}+B_{i}^{\prime}\right]+ \\
& k\left[R_{i}^{\prime} \times G_{i}^{\prime}+R_{i}^{\prime} \times B_{i}^{\prime}+G_{i}^{\prime} \times B_{i}^{\prime}\right]+ \\
& R_{i}^{\prime} \times G_{i}^{\prime} \times B_{i}^{\prime}-L^{3} \times C_{R_{i}, G_{i}, B_{i}}^{\prime \text { RCB }}=0 .
\end{aligned}
$$


where $R_{i}^{\prime}, G_{i}^{\prime}$, and $B_{i}^{\prime}$ stand for $R_{i}+1, G_{i}+1$, and $B_{i}+1$, respectively. We obtain the desired output triplet $\left(R_{o}, G_{o}, B_{o}\right)$, by solving this cubic equation in function of $k$, as the input one plus the displacement $k$, i.e., $\quad\left(R_{i}+\langle k\rangle, C_{i}+\langle k\rangle, B_{i}+\langle k\rangle\right)$, where $\langle k\rangle$ means the nearest integer to $k \in \mathbb{R}$. Equation 4 can be easily solved by [20] or by the classical Cardian's methods which use transcendental functions. We chose to use the former, since it is faster and mathematically simpler than the latter.

Our generic method hue-preserving [11], since any image pixel is enhanced following a shift transformation by a $k$ factor, i.e., from $\left(R_{i}, G_{i}, B_{i}\right)$ to $\left(R_{o}, G_{o}, B_{o}\right)=\left(R_{i}+k, G_{i}+k, B_{i}+k\right)$.

Having described this generic method, the next subsections show our variant method, which differ only on the histogram dimension used. By respecting the chronology's conception of our methods, the method based on $R G, R B$ and $G B$ 2D histograms [16] (from now on HP2DHE method), is described first in Section 4.2. Then, the method based on $1 D$ histograms [17] (from now on HP1DHE method) is presented in Section 4.3.

\subsection{D Hue-preserving Histogram Equalization}

In this section, our HP2DHE method is presented. It is important to note that it is initially introduced in [16]. It uses $2 D$ histograms (as defined in Section 2) and is based on the correlation of channels two-at-a-time to perform HE. The cumulative density function, $C^{I R G B}$, is computed as the product of the three $2 D$ cumulative functions for any entry $\left(R_{i}, G_{i}, B_{i}\right)$, i.e.,

$$
C_{R_{i}, G_{i}, B_{i}}^{I^{R C B}}=C_{R_{i}, G_{i}}^{I^{R C}} C_{R_{i}, B_{i}}^{I^{R B}} C_{G_{i}, B_{i}}^{I^{\text {GB }}} .
$$

We hypothesized that the three channels in an image are usually not simultaneously correlated. This is the main reason for calculating this pseudo-cumulative density function as the product of three $2 D$ cumulative density functions is.

Observe that Equation 2 is solved iteratively in [17], as done in [19] (TV3DHE method) as well, by using a non hue-preserving transformation. Here, we propose to use the hue-preserving shift transformation and the solution of Equation 2 described in the previous subsection modifying the method originally proposed in [17]. These two modifications on the HP2DHE method presented here reduces its time complexity from $O\left(\max \left(\operatorname{mnL}, L^{2}\right)\right)$ to $O\left(\max \left(m n, L^{2}\right)\right)$ and make it huepreserving

\subsection{Hue-preserving 1D Histogram Equalization}

In this section, a hue-preserving HE method based on the $R G B$ color space for IE is presented. It is also a variant of the generic method described in Section 4.1 and uses $1 D$ histograms. The independence assumption of color channels consists in the hypothesis of this method, and for computing the cumulative density function this assumption is used.

We use $1 D$ histograms to estimate a $3 D$ probability distribution function, and then equalize the conceived histogram through the estimated function. Hence, the function $C^{I R G B}$ is estimated for any entry $\left(R_{i}, G_{i}, B_{i}\right)$ as the product of every cumulative distribution $C_{R i}{ }^{I R}, C_{G i}{ }^{I G}$, and $C_{B i}{ }^{I B}$, following the rule, i.e., 


$$
C_{R_{i}, G_{i}, B_{i}}^{I^{R C B}}=C_{R_{i}}^{I^{R}} C_{G_{i}}^{I^{C}} C_{B_{i}}^{I^{B}}
$$

Note that, in Equation 6, $C^{I R G B}$ is defined with a true dimensional mathematical meaning, i.e., $C^{\prime R G B}$, a $3 D$ cumulative function, is computed as the product of three $1 D$ cumulative functions, while in Equation $5 C^{\prime I R G B}$ is defined with a false dimensional mathematical meaning, i.e., $C^{\prime I R G B}$ is computed as the product of three $2 D$ cumulative functions. Nevertheless, the images processed by the HP2DHE method produce similar results to the HP1DHE method, as the experiments reported in Section 5.

As we use $1 D$ histograms, this method has the time complexity greater than the HP $2 D H E$ method, i.e., $O(\max (\operatorname{mn}, L))$, and the space complexity is linear, i.e., $O(L)$. Furthermore, the space and time complexities of HP1DHE are the best to our knowledge, which are exactly the ones of the C1DHE method.

\section{EXPERIMENTS}

The majority of image enhancement methods found in the literature, including our previous works [16,17], assess the contrast improvement of the output image by comparing it to the original one. In $[16,17]$, we claimed that it is difficult to judge a processed enhanced image using a subjective assessment. Hence, in this work, we use quantitative measures to assess the original and processed images produced by the methods described in Section 3 and ours (presented in Section 4), and then perform an objective comparison among them. The measures used for comparing the methods are defined in Section 4.1. The numerical results obtained through these quantitative measures in a dataset of 300 images taken from the University of Berkeley [21] are analyzed and discussed in Section 4.2.

\subsection{Measures for Assessing Color Images Quality and Contrast}

In this section, two types of measures, which can be used to evaluate color images, are described. The first one is a color image quality measure (CIQM) [18], defined by using the image color naturalness and colorfulness indexes, and is used to verify if the HE methods preserve the quality of the images. The second measure aims to show how much the HE methods improve the contrast of the original image, and refers to the contrast in the CIELUV and in the RGB color spaces.

In order to define our first type of measure, we first need to calculate the color naturalness index $(C N I)$ and the colorfulness index $(C C I)$. These two indexes are defined in the CIELUV color space [18]. It is important to note that essential implementation details are not clear in [18], even though the conversions required for computing the CIQMs are said to be standard. Due to that, here, we present the required conversions in very high level of details.

The first index, the $C N I$, is computed as follows:

1. Converting the input image from the $R G B$ color space to the required CIELUV color space. This is done by first converting the image from the $R G B$ color space to the $X Y Z$ one (using $D_{65}$ white point), i.e., 
International Journal of Computer Science \& Information Technology (IJCSIT) Vol 4, No 5, October 2012

$$
\left[\begin{array}{l}
X \\
Y \\
Z
\end{array}\right]=\left[\begin{array}{lll}
0.4124 & 0.3576 & 0.1805 \\
0.2126 & 0.7152 & 0.0722 \\
0.0193 & 0.1192 & 0.9505
\end{array}\right] \times\left[\begin{array}{c}
t(R) \\
t(G) \\
t(B)
\end{array}\right],
$$

where

$$
t(K)= \begin{cases}((K+0.055) / 1.055)^{2.4}, & \text { if } K>0.04045 \\ K / 12.92, & \text { otherwise. }\end{cases}
$$

Having the image in the $X Y Z$ color space, we convert it to the CIELUV one, i.e.,

$$
\begin{gathered}
L^{*}=\left\{\begin{array}{cl}
116\left(Y / Y_{n}\right)^{1 / 3}-16, & \text { if } Y / Y_{n}>0.008856 \\
903.3\left(Y / Y_{n}\right), & \text { otherwise. }
\end{array}\right. \\
u^{*}=13 L^{*}\left(u^{\prime}-u_{n}^{\prime}\right), \\
v^{*}=13 L^{*}\left(v^{\prime}-v_{n}^{\prime}\right),
\end{gathered}
$$

where

$$
\begin{aligned}
& u^{\prime}=(4 X) /(X+15 Y+3 Z), \\
& v^{\prime}=(9 Y) /(X+15 Y+3 Z),
\end{aligned}
$$

and $u_{n}^{\prime}$ and $v_{n}^{\prime}$ are computed using the $D_{65}$ white point - $\left(X_{n}, Y_{n}, Z_{n}\right)=$ $(95.047,100.000,108.883)$, based on Equations 12 and 13.

2. Computing the hue $\left(H_{u v}{ }^{*}\right)$ and saturation $\left(S_{u v}{ }^{*}\right)$, i.e.,

$$
\begin{aligned}
& H_{u v}^{*}=\arctan \left(v^{*} / u^{*}\right), \\
& S_{u v}^{*}=C_{u v}^{*} / L^{*}=\sqrt{\left(u^{*}\right)^{2}+\left(v^{*}\right)^{2}} / L^{*} .
\end{aligned}
$$

3. Thresholding the $L^{*}$ and $S_{u v}{ }^{*}$ components, where $L^{*}$ values between 20 and 80 and $S_{u v}{ }^{*}$ values over 0.1 are kept.

4. Defining three kinds of pixels according to hue value $\left(H_{u v}{ }^{*}\right): 25-70$ is called "skin" pixels, $95-135$ is called "grass" pixels, and $185-260$ is called "sky" pixels, following the Yendrikhovskij's psychophysics studies [18]. Note that saturation and hue values are defined based on polar coordinates, and the hue varies from 0 to 360 degrees (see Figure 1).

5. Computing the averaged saturation values for "skin" $S_{\text {skin }}$, "grass" $S_{\text {grass }}$, and "sky" $S_{\text {sky }}$ pixels, i.e., respectively. 
International Journal of Computer Science \& Information Technology (IJCSIT) Vol 4, No 5, October 2012

6. Computing local $C N I$ values for "skin" $N_{\text {skin }}$, "grass" $N_{\text {grass, }}$, and "sky" $N_{\text {sky }}$ pixels:

$$
\begin{aligned}
N_{\text {skin }} & =\exp \left(-0.5\left(\left(\bar{S}_{\text {skin }}-0.763\right) / 0.524\right)^{2}\right), \\
N_{\text {grass }} & =\exp \left(-0.5\left(\left(\bar{S}_{\text {grass }}-0.810\right) / 0.528\right)^{2}\right), \\
N_{\text {sky }} & =\exp \left(-0.5\left(\left(\bar{S}_{\text {sky }}-0.432\right) / 0.221\right)^{2}\right) .
\end{aligned}
$$

The values $0.763,0.810,0.432,0.524,0.528$ and 0.221 were determined experimentally [18].

7. Finally, computing the global $C N I$ value:

$$
C N I=\frac{\left(n_{\text {skin }} N_{\text {skin }}+n_{\text {grass }} N_{\text {grass }}+n_{\text {sky }} N_{\text {sky }}\right)}{\left(n_{\text {skin }}+n_{\text {grass }}+n_{\text {sky }}\right)} .
$$

Note that the conversion described above is in low level of detail such that the numerical results presented in this section can be easily reproduced.

Our second index, the $C C I$, can be easily computed as

$$
C C I=\mu_{S_{u v}^{*}}+\sigma_{S_{u v}^{*}} \text {, }
$$

where ${ }_{S u v}{ }^{*}$ and $\sigma_{S u v}{ }^{*}$ stand for the mean and standard deviation of the saturation in CIELUV $S_{u v}{ }^{*}$, defined in Equation 15, respectively.

Having calculated these two indexes, we define the color image quality measure $Q$ in terms of $C N I$ and $C C I$, i.e.,

$$
Q=w \times C N I+(1-w) \times C C I / C C I_{\max },
$$

where the weighting parameter $w$ is set to 0.75 as suggested in [18], and $C C I_{\max }$ is set to 2.8396 following the maximum $C C I$ value found in our experiments. This first measure depicts the quality of both the processed and the original images in terms of color.

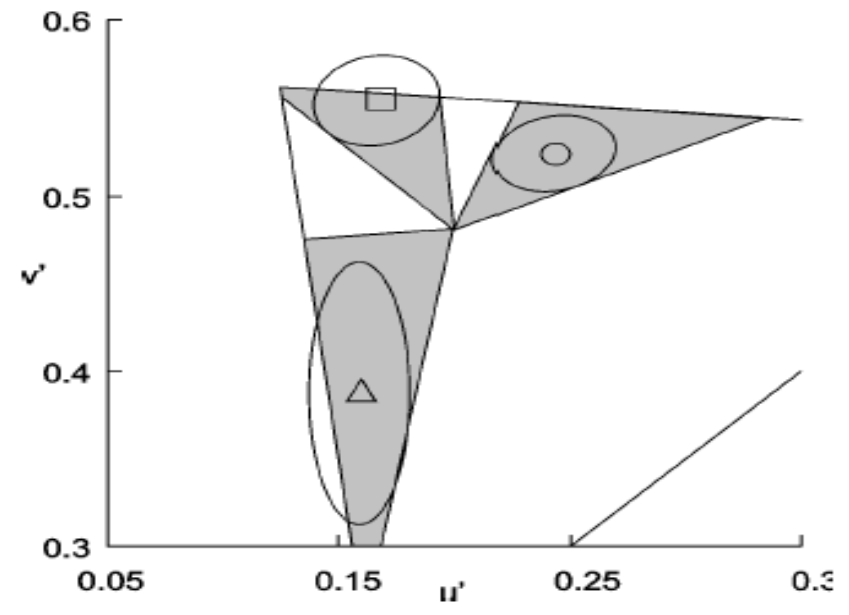

Figure 1. The "skin", "grass" and "sky" segments derived from the naturalness judgments of the colors [18], where the "skin", "grass" and "sky" segment centers are represented by a circle, a square, and a triangle, respectively, and the ellipses stand for standard deviations of a Gaussian approximation to subject's responses. Data are shown in the CIELUV color space. 
Now we define the measure of contrast. In order to do that, we first define the regional standard deviation of the luminance, i.e.,

$$
L_{\sigma}^{\alpha}(x, y)=\frac{\sqrt{\sum_{m=x-W}^{x+W} \sum_{t x=y-W}^{y+W}\left(L^{\alpha}(m, n)-L_{\mu}^{\alpha}(x, y)\right)^{2}}}{(2 W+1)^{2}},
$$

where

$$
L_{\mu}^{\alpha}(x, y)=\frac{1}{(2 W+1)^{2}} \sum_{m=x-W}^{x+W} \sum_{n=y-W}^{y+W} L^{\alpha}(m, n),
$$

and $L^{a}$ stands either for the luminance $L^{*}$ in the CIELUV color space (defined as in Equation 9), or the one in the $R G B$ color space, which can be defined as the average of the three channels $R, G$ and $B$, i.e., $L^{R G B}=\left(R_{i}+G_{i}+B_{i}\right) 3$, and the parameter $W$ is setup to 24 (i.e., blocks of $49 \times 49$ pixels as in [22]).

From here, we define the overall contrast of an image by the mean of the regional standard deviations of the luminance [22]. This measure provides a gross measure of the regional contrast variations, and it has been used by [23] as a measure of contrast in gray-level images.

Note that we define the contrast for the luminance in both the CIELUV and $R G B$ color spaces. In the CIELUV color space it is done because it is where the color quality image measure is defined, and in the $R G B$ color space because it is where our methods work. We do that to highlight that the HE methods improve the contrast for the luminance in both color spaces, as the analysis of the results, in the next section, will confirm.

\subsection{Computational Results}

This section presents and discusses the numerical results obtained by using the metrics described in the previous section to evaluate the two proposed methods (HP1DHE and HP2DHE) and the others described in Section 4 (C1DHE and TV3DHE) in a dataset composed of 300 images. We compute, for both the original and the processed images, the contrast in both the CIELUV and $R G B$ color spaces, as described in Equation 22. We also compute the CIQMs, as described in Equations 19, 20 and 21. Tables 1 and 2 show these data. Note that the values in both tables are presented in the form $\pm \sigma$, i.e., the mean and standard deviation of the measures computed on the dataset of 300 images. All images used in this experiment can be seen in [24]

Table 1. Contrast for the images in the CIELUV and RGB colors spaces.

\begin{tabular}{|l|c|c|}
\hline Method & $\boldsymbol{L}^{*}$ & $\boldsymbol{L}^{\boldsymbol{R} \boldsymbol{B} \boldsymbol{B}}$ \\
\hline Original & $12.53 \pm 15.86$ & $31.13 \pm 98.02$ \\
\hline C1DHE & $18.38 \pm 14.28$ & $47.11 \pm 95.21$ \\
\hline HP1DHE & $18.14 \pm 13.75$ & $46.73 \pm 92.33$ \\
\hline HP2DHE & $18.55 \pm 15.29$ & $47.02 \pm 100.20$ \\
\hline TV3DHE & $13.30 \pm 8.36$ & $36.44 \pm 59.64$ \\
\hline
\end{tabular}

The contrast in both the RGB and CIELUV color spaces for the processed and original images are shown in Table 1. From this table, we observe that the images processed by our methods, i.e., HP1DHE and HP2DHE, have the value of the contrast increased, in average, about $50 \%$ in both the CIELUV and $R G B$ color space. In a similar fashion increase the values of the contrast of images processed by the C1DHE method. On the other hand, the contrast of the processed images by the TV $3 D$ HE method is increased the less. Observe that, in general, the improvement of the value of contrast in the $R G B$ color space is proportional to the one in the CIELUV space (the 
range of the $R G B$ luminance is $[0,255]$ (with $L=256$ ) and the CIELUV luminance is $[0,100]$ ). Confirming what we had hypothesized in the previous section, the HE methods increased the contrast in both color spaces. From this first analysis, we state that significant increasing in the value of image contrast is yielded by our methods and the $\mathrm{C} 1 D \mathrm{HE}$ method.

Table 2. Color image quality measures.

\begin{tabular}{|c|c|c|c|c|c|c|c|c|}
\hline Method & \multicolumn{2}{|r|}{$Q$} & \multicolumn{3}{|c|}{ CNI } & \multicolumn{3}{|c|}{$C C I$} \\
\hline Original & 0.6754 & \pm 0.0195 & 0.8064 & \pm & 0.0332 & 0.8026 & \pm & 0.1234 \\
\hline C1DHE & 0.6780 & \pm 0.0141 & 0.7834 & \pm & 0.0260 & 1.0275 & \pm & 0.1329 \\
\hline HP1DHE & 0.6557 & \pm 0.0205 & .7829 & \pm & 0.0351 & 0.7779 & \pm & 0.0673 \\
\hline HP2DHE & 0.6673 & \pm 0.0230 & 0.7828 & \pm & 0.0408 & 0.9105 & \pm & 0.0987 \\
\hline TV3DHE & 0.5831 & \pm 0.0160 & 0.7197 & \pm & 0.0247 & 0.4923 & \pm & 0.0498 \\
\hline
\end{tabular}

Table 2 shows the $Q, C N I$ and $C C I$ measures for the original and processed images. Note that a weighting function of the $C N I$ and $C C I$ measures produces the $Q$ measure and the first numerical column in this table reports these values. It is important to note that, in average, the images processed by our methods have preserved the values of $Q$ in the processed images close to the value in the original ones. This means that the quality of the original images are similar to the ones produced by our methods. Also note that the images enhanced by our methods obtained similar $Q$ values to the ones obtained by the C1DHE method. On the other hand, the $Q$ values computed from the original images are quite larger than the ones produced from the image processed by the TV $3 D$ HE method. This shows that the images generated by the TV $3 D H E$ method are with deteriorated color quality.

The values for the $C N I$ measure is on the second numerical column of Table 2. We can observe that, in average, the $\mathrm{C} 1 \mathrm{DHE}$ and our methods keep the naturalness of the produced images close to the one from the original image, while the $C N I$ values from the original images are significantly larger than the ones obtained from the images produced by the TV $3 D$ HE method.

The values for the $C C I$ measure are reported on the third numerical column of Table 2. Note that the CCI measure is based on statistics from the CIELUV color space: the mean and standard deviation of the saturation of the image. From the results reported, we can see that, in average, the values of the $C C I$ measure from the original to the processed images is more frequently increased by the C1DHE method. Since it equalizes the three $R, G$ and $B 1 D$ histograms freely and separately, it is the one that achieves such result. In contrast, not being hue-preserving is the wellknown drawback of $\mathrm{C} 1 D \mathrm{HE}$ method. Such characteristic will be discussed and illustrated further in this section. In average, the $C N I$ and $C C I$ values and, consequently the $Q$ value, produced from the images generated by the TV $3 D$ HE method are not close to the values of the original images. The fact that the $C C I$ values obtained from the images generated by the TV $3 D H E$ method are quite different from the ones in the original images supports the hypothesis subjectively claimed in [16] and [17] that the images produced by the TV3DHE method are over enhanced or over saturated. In other words, the saturation values of the images produced by other methods are quite larger than the ones produced by the TV $3 D$ HE method.

From the analysis regarding the contrast and the CIQMs, we stated that: 1) Our methods produce images with contrast values that are in average $50 \%$ greater than the contrast of the original images, while the color quality, measured by the colorfulness and naturalness indexes, of the original image are close to the ones of the images generated by our methods; 2) The smaller improvement in the contrast of the original image is produced by the images generated by the TV $3 D$ HE method. Moreover, over enhanced images are produced by this method. Also these images have deteriorated color quality; 3) Considering the results for contrast enhancement and 
color quality preservation, the values achieved by our methods are as good as the ones for the C1DHE method.

Note that in order to make the TV $3 D$ HE method faster and hue-preserving, one can perform changes on it, by applying our shift hue-preserving transform. However, even after these modifications, the TV $3 D$ HE method would continue to produce images that are over enhanced and the contrast improvement would not be significant.

Although the $\mathrm{C} 1 D \mathrm{HE}$ method is six times faster than our methods and it presented good results in our numerical analysis, the $\mathrm{C} 1 D \mathrm{HE}$ is not suitable for real-world applications: this method does not preserve the hue of the original image in the images produced. As a result, the C1DHE method may produce images with have unnatural colors, even though the CNI, CCI and, consequently, $Q$, indicate that the original images have color quality close to the ones of the produced images. These conflicting results show that the CQIMs used in this work have a drawback. Although the color quality of an image by means of the naturalness and colorfulness indexes can be quantitatively represented by them, in such assessment these measures do not take into account at a the same time the processed and original images.

We will careful analyze one example of an image extracted from the 300 presented in the data base, named "landscape", in order to exemplify the conclusions reached. In Table 3, we present the contrast and the $C N I, C C I$ and $Q$ values for the original and processed landscape images shown in Figure 2. In Figure 2(b), it is quite noticeable the fact that the C1DHE method is not hue-preserving. We can see that, regarding the original image in Figure 2(a), the colors present in the image in Figure 2(b) look unnatural, even though the $C N I, C C I$ and $Q$ values of the original image are close to the ones from the processed image. In Figure 2(c), we also can see that the TV $3 D$ HE method produce an image which is over enhanced, i.e., the colors are over saturated, as previously explained in this section. Furthermore, from Table 3, we can also observe that the TV $3 D H E$ method generated an image in which the contrast increasing is the smallest among the compared methods.

Table 3. Color image quality and contrast measures for the images in Figure 2.

\begin{tabular}{|l|c|c|c|c|c|}
\hline \multirow{2}{*}{ Method } & \multicolumn{3}{|c|}{ Color Quality } & \multicolumn{2}{c|}{ Contrast } \\
\cline { 2 - 6 } & $\boldsymbol{Q}$ & $\boldsymbol{C N I}$ & $\boldsymbol{C C I}$ & $\boldsymbol{C I E L U V}$ & $\boldsymbol{R G B}$ \\
\hline Original & 0.7038 & 0.8540 & 0.7196 & 07.00 & 17.03 \\
\hline C1DHE & 0.7681 & 0.9292 & 0.8089 & 12.09 & 30.32 \\
\hline HP1DHE & 0.7210 & 0.8725 & 0.7575 & 11.50 & 28.98 \\
\hline HP2DHE & 0.6504 & 0.7688 & 0.8381 & 11.00 & 27.59 \\
\hline TV3DHE & 0.7140 & 0.9004 & 0.4392 & 08.76 & 23.68 \\
\hline
\end{tabular}




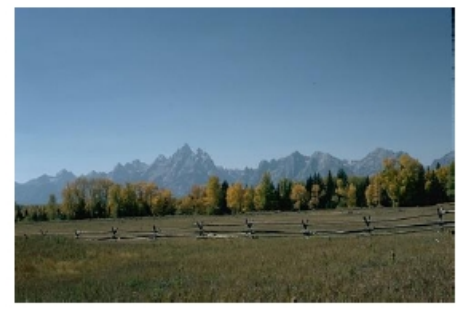

(a)

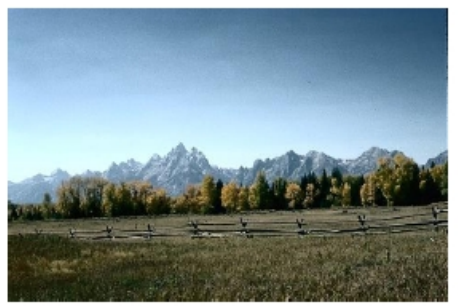

(d)

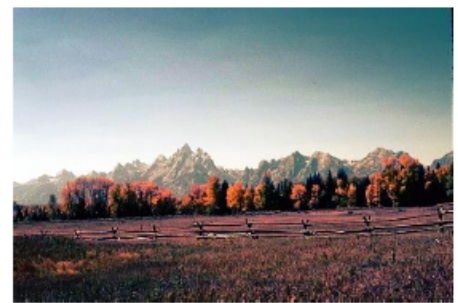

(b)

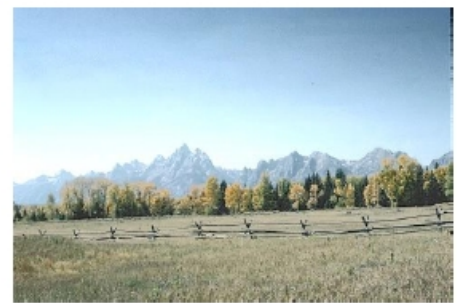

(c)

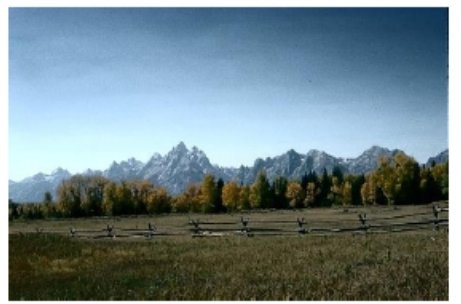

(e)

Finally, the claims about our methods are verified in the images in Figures 2(d) and 2(e) and confirmed in Table 3. As observed, the images have their contrast value increased by, in average, $50 \%$, while their color quality measures are kept close to the ones of the original image. Moreover, recall that our methods are hue-preserving.

\section{Conclusions}

Two fast hue-preserving HE methods based on $1 D$ and $2 D$ histograms of the $R G B$ color space for image contrast enhancement are proposed in this work. The HP1DHE and HP2DHE methods have $O(\max (m n, L))$ and $O\left(\max \left(m n, L^{2}\right)\right)$ time complexities, respectively. As the term $m n$ on the time complexity expression is much more important than the second term $\left(L L^{2}\right)$, on a run-time comparison the methods have similar performance. Moreover, the HP1DHE and HP2DHE methods are linear and quadratic for space complexity, i.e., $O(L)$ and $O\left(L^{2}\right)$, respectively. Hence, such time and space complexities comply with real-time application requirements.

Although our method is six times slower the C1DHE method, it is not hue preserving. As shown in our experiments (Section 5), the processed images by the C1DHE method might look unrealistic. Remark that our methods are about ten times faster than the TV $3 D H E$ one. In practice, our methods enhance $512 \times 512$ image pixels in 100 milliseconds on a Pentium 4 $2 \mathrm{GHz}$.

We evaluated the resulting images objectively by using measures of contrast, naturalness and colorfulness [18] on a dataset composed of 300 images, such that a quantitative comparison could be performed. The experiments showed that our method produces images whose value of the contrast is in average $50 \%$ greater than the one of the original image. They also showed that the quality of image in terms of both naturalness and colorfulness produced by our methods are close to the quality of the original image. The C1DHE method also achieves similar results. However, this classical method does not preserve the hue and produce images that are not realistic with respect to the original image.

For future works, we plan to compare our methods based on the $R G B$ color space with other ones designed on a real-time application framework and based on other color spaces. The comparison should be based on the contrast improvement and the quality of the processed images, but run- 
time should be taken into account as well. The drawbacks pointed on the color quality measures should also be considering. We plan to modify the current measures by taking into account the original and processed images simultaneously.

\section{ACKNOWLEDGEMENTS}

This work was supported by French Government grant ANR SURF NT05-2_45825 and by the CNPq/MCT and CAPES-COFECUB/MEC, Brazilian Government's research support agencies.

Due to the financial providing support during part of his stay in France, where a substantial part of this work was developed, the first author is very grateful to Prof. Dr Hugues Talbot. The authors would like to thank Prof. Dr. G.L. Pappa for reading the first drafts of this paper.

\section{REFERENCES}

[1] J.-H. Han, S. Yang, and B.-U. Lee, "A Novel 3-D Color Histogram Equalization Method With Uniform 1-D Gray Scale Histogram,” IEEE Transactions on Image Processing, vol. 20, no. 2, pp. 506-512, Feb. 2011.

[2] K. Sreedhar, and B. Panlal, "Enhancement of images using morphological transformations," International Journal of Computer Science \& Information Technology (IJCSIT), vol. 4, no. 1, pp. 3350, Feb 2012.

[3] T. Celik, and T. Tjahjadi, "Automatic Image Equalization and Contrast Enhancement Using Gaussian Mixture Modeling," IEEE Transactions on Image Processing, vol. 21, no. 1, pp.145-156, 2012.

[4] Divakaran, "Spectral Analysis of Projection Histogram for Enhancing Close matching character Recognition in Malayalam," International Journal of Computer Science \& Information Technology (IJCSIT) Vol 4, No 2, April 2012

[5] D. Menotti, L. Najman, J. Facon, and A. Araújo, "Multi-histogram equalization methods for contrast enhancement and brightness preserving," IEEE Transaction on Consumer Electronics, vol. 53, no. 3, pp. 1186-1194, Aug. 2007.

[6] M. Kaur, J. Kaur, J. Kaur, "Survey of Contrast Enhancement Techniques based on Histogram Equalization," International Journal of Advanced Computer Science and Applications (IJACSA), vol. 2, no. 7, 2011.

[7] J. Han, S. Yang, and B. Lee, "A novel 3-d color histogram equalization method with uniform 1-d gray scale histogram,” IEEE Transactions on Image Processing, vol. 20, no. 2, pp. 506-512, Feb. 2011.

[8] E. Vahedi, R. Zoroofi, and M. Shiva, "Toward a new wavelet-based watermarking approach for color images using bio-inspired optimization principles," Digital Signal Processing, vol. 22, no. 1, pp. 153162, Jan. 2012.

[9] R. Gonzalez and R. Woods, Digital Image Processing, 3rd ed. Prentice Hall, Jan. 2009.

[10] R. Berns, F. Billmeyer, and M. Saltzaman, Billmeyer and Saltzaman's Principles of Color Technology, 3rd ed. New York, USA: Wiley, 2000.

[11] S. Naik and C. Murthy, "Hue-preserving color image enhancement without gamut problem," IEEE Transactions on Image Processing, vol. 12, no. 12, pp. 1591-1598, Dec. 2003.

[12] I. Bockstein, "Color equalization method and its application to color image processing," Journal of the Optical Society of America, vol. 3, no. 5, pp. 735-737, 1986.

[13] R. Strickland, C. Kim, and W. McDonel, "Digital color image enhancement based on the saturation component," Optical Engineering, vol. 26, no. 7, pp. 609-616, 1987.

[14] B. Williams, C. Hung, K. Yen, and T. Coleman, "Image enhancement using the modified cosine function and semi-histogram equalization for gray-scale and color images," in International Conference on Systems, Man and Cybernetics, vol. 1. IEEE, 2001, pp. 518-523.

[15] E. Pichon, M. Niethammer, and G. Sapiro, "Color histogram equalization through mesh deformation," in Proceedings of International Conference on Image Processing (ICIP), vol. 2. IEEE, 2003, pp. $117-$ 120.

[16] D. Menotti, A. Melo, A. Araújo, J. Facon, and E. Sgarbi, "Color image enhancement throught 2D histogram equalization," in 13th International Conference on Systems, Signals and Image Processing (IWSSIP 2006), Budapest, Hungry, 21-23 September 2006, pp. 235-238. 
[17] D. Menotti, L. Najman, A. Araújo, and J. Facon, "A fast hue-preserving histogram equalization method for color image enhancement using a bayesian framework," in 14th International Conference on Systems, Signals and Image Processing (IWSSIP 2007), Maribor, Slovenie, 27-30 June 2007, pp. 433-436.

[18] S. Yendrikhovskij, F. Blommaert, and H. de Ridder, "Perceptually optimal color reproduction," in Proceedings of the Human Vision and Electronic Imaging III, vol. 3299. SPIE, 1998, pp. 274-281.

[19] P. Trahanias and A. Venetsanopoulos, "Color image enhancement through 3-d histogram equalization," in Proceedings of 11th International Conference on Pattern Recognition (ICPR). IEEE, 1992, pp. 545-548.

[20] R. Nickalls, “A new approach to solving the cubic: Cardan's solution revealed," The Mathematical Gazette, vol. 77, pp. 354-359, 1993.

[21] D. Martin, C. Fowlkes, D. Tal, and J. Malik, "A database of human segmented natural images and its application to evaluating segmentation algorithms and measuring ecological statistics," in Proc. 8th International Conference on Computer Vision, vol. 2, July 2001, pp. 416-423.

[22] D. Jobson, Z. Rahman, and G. Woodell, "The statistics of visual representation," in Proceeding of SPIE: Visual Information Processing XI, vol. 4736. SPIE, 2002, pp. 25-35. [Online]. Available: http://citeseer.ist.psu.edu/598477.html

[23] K.-Q. Huang, Z.-Y. Wu, and Q. Wang, "Image enhancement based on the statistics of visual representation," Image and Vision Computing, vol. 23, pp. 51-57, 2005.

[24] D. Menotti, “Test image," 2012, http://www.decom.ufop.br/menotti/FHPHEM/..

\section{Authors}

David Menotti was born in October 1978, Andirá, Paraná, Brazil. He received his Computer Engineering and Informatics Applied Master degrees from Pontifícia Universidade Católica do Paraná (PUCPR), Brazil, in 2001 and 2003, respectively. In 2008, he received his cotutelle $\mathrm{PhD}$ degree in Computer Science from both the Universidade Federal de Minas Gerais (UFMG), Belo Horizonte, Brazil and the Université Paris-Est/Groupe ESIEE, Paris, France. In 2009, he won two awards for advising undergraduate works in Brazilian Workshops. He is with the Computing Department (DECOM), Universidade Federal de Ouro Preto (UFOP) since 2008 as an Associate Professor. And currently, he is working as colaborator professor at both Post-Graduate Program of Computer Sciente, Computer Science Departament (DCC), UFMG and Post-Graduate Program of Applied Informatics, PUCPR. His research interests include image processing, pattern recognition, computer vision and algorithms for information retrieval systems.

Laurent Najman received the Habilitation à Diriger les Recherches from the Université Paris-Est, Marne-la-Vallée, France, in 2006, the Ph.D. degree in applied mathematics (Félicitations du Jury) from the Université Paris Dauphine, Paris, France, in 1994, and the Ing. degree from the Ecole des Mines de Paris in 1991. He was with the Central Research Laboratories of Thomson-CSF for three years, working on problems of infrared image segmentation using mathematical morphology. He then joined a start-up company, Animation Science, in 1995, as Director of Research and Development. In 1998, he joined OCÉ Print Logic

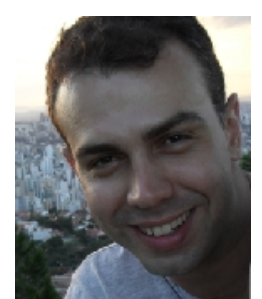
Technologies as a Senior Scientist, where he worked on various problem of image analysis dedicated to scanning and printing. Since 2002, he is with the A2SI Laboratory, ESIEE, Paris, where is Professor and also a member of the Institut Gaspard Monge. His current research interest is in discrete mathematical morphology 
Jacques Facon received the Ph.D. degree in System Control from the UTC Université de Technologie, Compiègne, France, in 1987. He was a Visiting Professor at the Departamento de Engenharia Elétrica e Informática Industrial, Universidade Tecnológica Federal do Paraná, Curitiba, Brazil, 1988-1997, an Associate Professor at the Departamento de Informática, Pontifícia Universidade Católica do Paraná, Curitiba, Brazil, 1997-2003. Since 2003, he has been a Full Professor at the Programa de PósGraduação em Informática Aplicada, Pontifícia Universidade Católica do Paraná, Curitiba, Brazil. His research interests include digital image processing, mathematical morphology and computer vision applications.

Arnaldo de Albuquerque Araújo received his B.Sc., M.Sc. and D.Sc. degrees in Electrical Engineering, from the Universidade Federal da Paraiba (UFPB), Brazil, in 1978, 1981 and 1987, respectively. Arnaldo is currently an Associate Professor at the Departamento de Ciência da Computação (DCC), Universidade Federal de Minas Gerais (UFMG), Belo Horizonte-MG, Brazil (since 1990). He was a Visiting Researcher at the Department d'Informatique, Groupe ESIEE Paris, France, 1994-1995, an associate professor at the Departamento de Engenharia Elétrica, UFPB, 1985-1989, a Research Assistant at the Rogowski-Institut, Technische Hochschule Aachen,

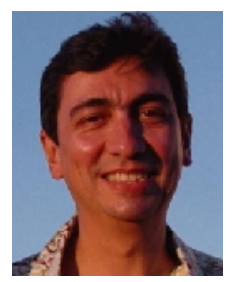
Germany, 1981-1985, and an Assistant Professor at DEE/UFPB, 1978-1985. He is the header of the Núcleo de Processamento Digital em Imagens and coordinator of the international cooperation agreement between UFMG and the French Schools ESIEE Paris and ENSEA Cergy-Pontoise. His research interests include digital image processing and computer vision applications to medicine, fine arts, and content based information retrieval. 